\title{
Análisis de reportajes publicados por el diario The New York Times en redes sociales sobre la realidad de América Latina en 2017
}

\author{
Analysis of reports published by The New York Times in social networks about the reality of Latin
}

America in 2017

Carlos Ricardo Gonzales García'

RECIBIDO: 17 DE ABRIL DE 2020

ACEPTADO: 25 DE JUNIO DE 2020

\section{RESUMEN}

La presente investigación aborda el tratamiento informativo, interpretativo y opinativo que un importante e influyente medio internacional, The New York Times (edición en español), brindó sobre la realidad de América Latina a través de sus reportajes durante 2017. Para tal efecto, se hizo uso del esquema a nivel superestructural desarrollado por Teun van Dijk (1990) y de los juicios interpretativos y categóricos propuestos por Santamaría y Casals (2000) para analizar las historias publicadas por dicho diario en Internet a través de sus redes sociales. Entre los

\footnotetext{
1 Maestro en Periodismo y Comunicación Multimedia, Magíster en Investigación de la Comunicación, Licenciado en Ciencias de la Comunicación, Docente, Universidad Nacional Mayor de San Marcos, Perú; carlos.gonzales1@unmsm.edu.pe, ORCID https://orcid.org/0000-0001-9408-4329 Google Scholar: https://bit.ly/2BNX5H7
}

Socialium revista científica de Ciencias Sociales, Vol 4 - No. 2, julio - diciembre 2020, pág. 1-10. 
principales hallazgos, se encontró que este periódico destaca, a profundidad, el esfuerzo heroico de los latinoamericanos por la pacificación interna en determinadas zonas de conflicto, tanto de México como de Colombia. Asimismo, se vale de testimonios personales para denunciar casos de espionaje y medio ambiente.

Palabras clave: periodismo, América Latina, reportajes, análisis de contenido

\section{ABSTRACT}

This research addresses the informative, interpretative and opinionated treatment that an important and influential international media, The New York Times (Spanish edition), provided about the reality of Latin America through its reports during 2017. To this end, it made use of the superstructure-level scheme developed by Teun van Dijk (1990) and the interpretative and categorical judgments proposed by Santamaría and Casals (2000) to analyze the stories published by the newspaper on the Internet through its social networks. Among the main findings, it was found that this newspaper highlights, in depth, the heroic effort of Latin Americans for internal peace in certain conflict zones, both in Mexico and Colombia. It also uses personal testimonies to denounce cases of espionage and the environment.

Keywords: journalism, Latin America, reports, media content

\section{Introducción}

Tratar la estructura periodística, obliga a estudiar las formas que adoptan los discursos acerca de los hechos de la actualidad y las unidades redaccionales en general. Por una parte, tenemos las distintas formas con que cuentan los mensajes para su expresión, como son, para nuestro interés, la interpretación y la opinión. Por la otra, la que corresponde a la organización interna del relato a través de estructuras piramidales (el esquema convencional típico) o categorías de nivel superestructural propuestas por el esquema de van Dijk, y que más adelante explicaremos.

En principio, ese atender a la forma de un discurso se torna significativa cuando, a través de los géneros discursivos, hallamos tipos textuales que dependen de ciertas 
situaciones de comunicación, a saber: la narración, la descripción, la argumentación, la exposición, entre otras.

Estas estructuras, a su vez, se corresponden con actitudes de tipo informativo (expositiva como noticias y reportajes cortos), interpretativo (descriptiva y narrativa como crónicas y reportajes en profundidad) y opinativa (argumentativa como cualquiera de los artículos de un periódico: editoriales, columnas, críticas de arte, etc.).

Sin embargo, de acuerdo a la teoría de la hibridación de géneros, referida por los profesores José María Casasús y Luis Núñez Ladevéze (Velásquez Ossa, 2011), la interpretación, entendida como intención persuasiva de su creador, puede encontrarse en textos informativos. Ese mestizaje de géneros también se puede advertir en textos como la crónica, ya que esta puede contar con la inclusión de opiniones o evaluaciones por parte del autor.

Si la interpretación, como refieren Santamaría y Casals (2000) llega a ser una parte fundamental de las noticias y los fragmentos de comentarios, evaluaciones y reacciones de los hechos realizados por el periodista se incluyen en crónicas y otros géneros (Dellamea, 1995) las cualidades de la objetividad (explicación) y subjetividad (opinión) nos llevarían a examinar la naturaleza de los juicios que utilizan. Santamaría y Casals hacen una diferenciación entre los juicios de textos interpretativos y los que pertenecen a los de tipo argumentativo. Así, los primeros, más orientados a lo objetivo, se disgregan en analíticos o apriorísticos, que ofrecen datos contextualizadores y antecedentes; sintéticos o a posteriori, los cuales intentan predecir ciertas realidades; hipotéticos y; disyuntivos o alternativos cuando plantean dos opciones. Por el lado de los opinativos, tenemos los categóricos que se dividen en juicios de hechos o de realidades; de intención; y de valor. Este último, a diferencia de los dos anteriores, no suele utilizar apreciaciones bastante subjetivas o ideológicas, sino básicamente juicios de hechos, personas y situaciones que refieren valores jerarquizados: políticos, sociales, éticos, religiosos (Santamaría y Casals, 2000). En cuanto a la estructura organizacional, por tradición, se proponen tres estructuras básicas de textos que determinan la entrada, el cuerpo y el final de todo género: pirámide normal o relato cronológico; pirámide invertida constituida por la fórmula 
de las 7Wh: quién (who), qué (what), a quién (who), dónde (where), cuándo (when), cómo (how) y por qué (why); y la estructura de rectángulos iguales, utilizada para obtener segmentos de textos de similar valor relativo. Por supuesto, existen otras estructuras como las mixtas (yunque o martillo, copa, reloj de arena, etc.).

No obstante, las diferentes partes que conforman las estructuras de un texto podrían comprenderse y jerarquizarse mejor si se las representan como discursos. Al respecto, Teun van Dijk (1990) propone que el discurso periodístico se pueda regir por un conjunto de categorías propias que posibilite la realización de los textos noticiosos, así como de crónicas y opiniones como editoriales y columnas (Dellamea, 1995). Estas categorías son el resumen (la macroproposición semántica), conformado por el titular (antetítulo, título, subtítulo) y el encabezamiento (copete y entradas). A través de esta macroestructura, la audiencia puede leer y comprender las noticias, pues se informa, aunque superficialmente, de los contenidos más destacados del texto (orden no cronológico).

En el esquema de van Dijk, una vez presentado el resumen, se desarrolla el texto a través del relato que está compuesta por la situación, que reúne un conjunto de sucesos que ocurrieron posiblemente en distintos escenarios, pero que son presentados de acuerdo a su relevancia. Esta categoría asimismo contempla las consecuencias del acontecimiento. Otro elemento que constituye la situación informativa es el antecedente, el cual diferencia dos tipos: el contexto, que denota la situación actual y la historia, que trata la evolución histórica, no reciente, de lo actual.

Otra categoría central presente en géneros informativos e interpretativos es el comentario, que incluye fragmentos opinativos, evaluativos a través de juicios de hechos, intención o de valor provenientes no solo de los protagonistas sino también de los propios periodistas.

El presente trabajo se enmarca en la tradición de aquellos estudios que se han preocupado de conceptualizar a América Latina y sus problemas a través de discursos y corrientes, fundamentalmente desde sectores populares organizados y movimientos sociales (Loza, 2012). Sin embargo, nuestro tema no ha sido muy trabajado desde el periodismo, menos desde una propuesta que integre en el análisis del discurso periodístico el estudio de lo informativo, interpretativo y juicioso en las 
historias y reportajes de un medio de alcance global. Tomando en cuenta este vacío en los estudios acerca del mensaje periodístico, formulamos la siguiente pregunta: $\dot{\imath}$ El periodismo que representa en Internet The New York Times en español se funda en el análisis de causas y consecuencias para comprender la complejidad y la diversidad de la realidad de América Latina?

\section{Método}

En primer lugar, se consultaron los libros y los artículos escritos por académicos como Teun van Dijk, Luisa Santamaría y María Jesús Casals, entre otros, quienes escribieron sobre las categorías del esquema periodístico y los juicios que los periodistas han utilizado en sus textos interpretativos y argumentativos. Luego se revisaron los textos que sobre la realidad de América Latina fueron publicados por The New York Times en internet durante 2017. El método tomó en cuenta el enfoque cuantitativo. Al respecto, hicimos una medición estadística de las categorías del discurso periodístico (propuesto por van Dijk) y de los juicios interpretativos y editorializantes (propuestos por Santamaría y Casals) que llegaron a concretarse en los textos del diario de referencia. La técnica empleada fue la del análisis de contenido.

\section{Resultados}

Temática. En el estudio se han registrado un total de 25 textos periodísticos (acompañados de 220 fotografías y siete videos) que trataron sobre distintos hechos en América Latina: salud, belleza, lenguaje y deportes fueron los que concentraron el $40 \%$ de la cobertura, seguido de temáticas donde suelen participar, además de ciudadanos, actores sociales de alta jerarquía (políticos y científicos). Entre estas tenemos las siguientes: desastres naturales, cambio climático y medio ambiente (28 $\%)$.

Países. Del conjunto de trabajos, el 57 \% estuvo concentrado en México, el $13 \%$ en Colombia y un $30 \%$ en el resto de países de la región, principal sudamericana (Brasil, Perú, Ecuador, Chile, Venezuela, Puerto Rico y Honduras).

Superestructura noticiosa. Basándonos en la macroestructura del discurso de van Dijk, 
hemos encontrado que un $96 \%$ de las notas cuentan con antecedentes, un $92 \%$ con historia, un $84 \%$ con consecuencias y un $100 \%$ con reacciones verbales. Sobre la categoría resumen, hemos hallado que el $76 \%$ presenta titulares de tipo interpretativo. Por otra parte, el $56 \%$ de titulares cuentan con algún complemento (mayoritariamente subtítulos). Los tipos de lead o entrada constituían sumarios más enfocados al qué y al quién (72 \%) que al cuándo o dónde (24\%). Finalmente, entre los leads especiales más destacados tenemos el conocido como relieve (84 \%).

Juicios. En cuanto a la naturaleza interpretativa y opinativa de estos materiales periodísticos, un $92 \%$ estaba conformado exclusivamente por juicios interpretativos (87\% sintético, $9 \%$ analítico y $4 \%$ hipotético).

\section{Discusión}

Desde la perspectiva de la teoría de la mediación, la cobertura periodística de América Latina de este siglo se ha manejado dentro dos criterios. Uno, cognitivo; el otro, en el orden estructural expresivo.

El primero de estos razonamientos, que podemos calificar de ideológico y tensional, ocurrió, por ejemplo, cuando emergieron en Sudamérica gobiernos de izquierda, algunos de ellos de corte populista, como los de Hugo Chávez (1999), Ricardo Lagos (2000), Luiz Inácio Lula da Silva y Néstor Kirchner (2003), Tabaré Vázquez (2005), Evo Morales (2006) y Rafael Correa (2007), acapararon "numerosos episodios públicos y subterráneos de conflicto con la prensa" (Kitzberger, 2009).

Esta tensión se explica porque entre la producción y difusión periodística de estos hechos y la asunción y reproducción de un sistema de valores, generados en la década de los noventa, el giro hacia la economía de mercado se supone había preparado el terreno para asumir en el siglo XXI el poder del capitalismo como el único sistema que reconoce «la naturaleza "racional" del ser humano, y, por tanto, la "libertad", como exigencia de estan, tal como lo sostuvo la escritora y filósofa, Ayn Rand, en su obra What is Capitalism?, publicado en 1967.

En ese aspecto, los medios de comunicación, en definitiva, mediadores (Gomis, 2008), asumen un ethos profesional que busca como poder preservar normas, valores, justificaciones de un tipo de modelo que puede pregonar o no, por ejemplo, 
la supremacía del mercado libre y el descrédito de la participación del Estado en la actividad económica.

En cuanto a lo estructural, los medios como titulares en la vida social, no tienen otra alternativa que ajustar el universo sociocultural del mundo que les rodea a un tratamiento que obedezca al modelo mediador que estos han construido en base a las capacidades de la técnica (géneros o esquemas periodísticos) y de la organización que forma (páginas o secciones) y distribuye los productos informativos. Interpretar la sociedad en dicho modelo significaría, en consecuencia, "remitir esas mismas prácticas a las necesidades del sistema de producción" (Martín Serrano, 1985).

El conflicto entre la apertura del medium al acontecer imprevisto, y su cerramiento en una forma comunicativa, pide una mediación estructural. La mediación estructural está destinada a conseguir que aquello que irrumpe sirva para realimentar las modalidades comunicativas de cada medio productor. Es una tarea agónica, en la que el medio se ve obligado a recurrir, para perpetuar su forma expresiva, a aquello mismo que cada día vulnera los marcos formales de la comunicación, es decir, el acontecimiento imprevisible. (Martín Serrano, 1986: 143).

La abundancia de juicios interpretativos sobre América Latina, en The New York Times, nos lleva a exponer, dentro de los parámetros de investigación que estudian los fenómenos que posibilitan la construcción de imaginarios sociales (realidad), la teoría del framing, unas de las más estudiadas, pero también uno de los sistemas más dispersos en definición de conceptos (Entman, 1993).

Desde su aparición, en el campo de la psicología, lvego de su paso por la sociología, hasta llegar a la comunicación y las políticas públicas, la teoría del framing se ha encontrado siempre con barreras conceptuales que imposibilitan su desarrollo con una base sólida y líneas de investigación bien definidas (Orozco, 2017).

En ese contexto disperso, la teoría del framing o del encuadre, se ha hecho camino en la búsqueda por entender las estructuras previas, el proceso y las consecuencias que trae consigo la construcción de fenómenos sociales, expresados en los discursos 
8

noticiosos. No está de más decir que, el framing, en una de sus definiciones más simples "seleccionar algunos aspectos de la realidad percibida y hacerlos más prominentes en un texto comunicativo, de forma que promuevan una definición del problema, una causa, una evaluación moral y una recomendación" (Entman, 1993), sigue siendo un enfoque que se va expandiendo y a la vez complejizando, no solo por el interés de unificar su definición, sino también por consensuar su metodología, la cual-por muchos años- se ha venido empleando desde el tradicional análisis de contenido hasta las técnicas del análisis del discurso.

En América Latina, el hecho de que la cobertura temática, mas no episódica, del diario neoyorkino se haya concentrado en países como México y, en menor grado, Colombia, es un síntoma cuya causa plantearía, según Tuchman (1978), en preguntarnos cómo el uso de los encuadres de los medios de comunicación transita por una comprensión del contexto y las actitudes de los periodistas, quienes son los responsables de concebir el hecho noticioso mediante el uso del discurso.

Asimismo, queremos señalar que el hecho de que temas vinculados a salud, belleza, lenguaje y deportes merecieran más tratamiento que problemas globales como los regímenes de gobierno (el populismo), la crisis económica, el cambio climático y los desastres naturales, nos lleva a evaluar, para un futuro trabajo, cómo trabaja una determinada organización a través de sus rutinas profesionales para que, dentro de un contexto simbólico determinado, sea consentido por su audiencia, cultural y socialmente (Sádaba, 2008).

Por otro lado, partiendo de la premisa que los discursos noticiosos carecen de objetividad, el framing ha venido aportando en el esclarecimiento del tratamiento informativo, sobre todo, en temas de relevancia política e ideológica. Por lo tanto, se concluye que "el trabajo de encuadre conceptual e ideológico para temas políticos posiciona los significados dominantes en el interior del contenido y presenta el acontecimiento" (Rodríguez, 2015).

\section{Conclusiones}

The New York Times, en español, hace una interpretación de la realidad de América Latina desde una postura ideológica camuflada mayormente en reportajes y crónicas de países como México y Colombia. En ella, pone en evidencia el esfuerzo 
que hacen heroicamente algunas personas por la pacificación en zonas de guerras internas.

Asimismo, denuncia a través del testimonio de distintos personajes, casos de espionaje o la destrucción del medio ambiente.

Sin embargo, el diario neoryorkino valora mayormente temas relacionados a salud (cesáreas, comida chatarra, bebidas azucaradas, obesidad, entre otros) así como algunos más relacionados a actividades deportivas, belleza y lenguaje.

\section{Referencias bibliográficas}

Brewer-Carías, A. R. (2000). Reflexiones críticas sobre la Constitución de Venezuela de 1999. Constitucionalismo iberoamericano en el Siglo XXI, 171-194.

Dellamea, A. (1995). El discurso informativo. Buenos Aires: Fundación Universidad a Distancia "Hernandarias".

Entman, R. M. (1993). Framing: Toward clarification of a fractured paradigm. Journal of communication, 43(4), 51-58.

Gomis, L. (2008). Teoría de los géneros periodísticos. Barcelona: UOCpress.

Kitzberger, P. (2009). Las relaciones gobierno-prensa y el giro político en América Latina. POSTData, 14(2), 157-181.

Martín Serrano, M. (1985). El estructuralismo antropológico y el mito de la postmodernidad. Los Cuadernos del Norte: Revista cultural de la Caja de Ahorros de Asturias, 6(29), 4-10.

Martín Serrano, M. (1986). La mediación de los medios de comunicación. Sociología de la Comunicación de Masas I. Escuelas y Autores, 141-162.

Orozco, R. (2017). Revisión de la teoría del framing: Análisis del conflicto sirio en el sistema mediático español (tesis de maestría). Universidad de Barcelona.

Rand, A. (1964). ¿Qué es el Capitalismo? Encyclopedia Britannica, 839-845.

Rodríguez, C. (2014). La teoría del framing aplicada al 'rescate'a España. MARCO (Márketing y Comunicación Política), 1

Sádaba, T. (2008). Framing: el encuadre de las noticias: el binomio terrorismo-medios. Buenos Aires: La Crujía Ediciones. 
10

Santamaría, L. \& Casals, M. J. (2000). La opinión periodística. Argumento y géneros para la persuasión. Madrid: Fragua.

Tuchman, G. (1978). Making news: A study in the construction of reality.

Van Dijk, T. A. (1990). La noticia como discurso: comprensión, estructura y producción de la información. Barcelona: Paidós

Velásquez Ossa, C. M. (2011). Una aproximación a los géneros periodísticos. En: V. M. García \& L. M. Gutiérrez (editores) (2011). Manual de géneros periodísticos. Bogotá: Ecoe Ediciones y Universidad de la Sabana. 